\title{
Journal of clinical monitoring and computing 2016 end of year summary: monitoring cerebral oxygenation and autoregulation
}

\author{
Thomas W. L. Scheeren ${ }^{1} \cdot$ Bernd Saugel $^{2}$
}

Received: 3 January 2017 / Accepted: 3 January 2017 / Published online: 24 January 2017

(C) The Author(s) 2017. This article is published with open access at Springerlink.com

\begin{abstract}
In the perioperative and critical care setting, monitoring of cerebral oxygenation $\left(\mathrm{ScO}_{2}\right)$ and cerebral autoregulation enjoy increasing popularity in recent years, particularly in patients undergoing cardiac surgery. Monitoring $\mathrm{ScO}_{2}$ is based on near infrared spectroscopy, and attempts to early detect cerebral hypoperfusion and thereby prevent cerebral dysfunction and postoperative neurologic complications. Autoregulation of cerebral blood flow provides a steady flow of blood towards the brain despite variations in mean arterial blood pressure (MAP) and cerebral perfusion pressure, and is effective in a MAP range between approximately $50-150 \mathrm{mmHg}$. This range of intact autoregulation may, however, vary considerably between individuals, and shifts to higher thresholds have been observed in elderly and hypertensive patients. As a consequence, intraoperative hypotension will be poorly tolerated, and might cause ischemic events and postoperative neurological complications. This article summarizes research investigating technologies for the assessment of $\mathrm{ScO}_{2}$ and cerebral autoregulation published in the Journal of Clinical Monitoring and Computing in 2016.
\end{abstract}

Keywords Monitoring - Tissue oxygenation - Cerebral blood flow $\cdot$ Autoregulation - Near infrared spectroscopy Cerebral oximetry

\section{Thomas W. L. Scheeren}

t.w.l.scheeren@umcg.nl

1 Department of Anesthesiology, University Medical Center Groningen, University of Groningen, Groningen, The Netherlands

2 Department of Anesthesiology, Centre of Anesthesiology and Intensive Care Medicine, University Medical Centre Hamburg-Eppendorf, Martinistrasse 52, 20246 Hamburg, Germany

\section{Introduction}

In the perioperative setting, particularly in patients undergoing cardiac surgery, monitoring of cerebral oxygenation $\left(\mathrm{ScO}_{2}\right)$ enjoys increasing popularity in recent years. The rationale behind its use is the attempt to early detect cerebral hypoperfusion, which may be caused by systemic hypotension or the use of the cardiopulmonary bypass, and thereby prevent cerebral dysfunction and postoperative neurologic complications [1]. In addition to the widespread use in cardiac anaesthesia and postoperative care, $\mathrm{ScO}_{2}$ monitoring has spread over the whole range of perioperative and critical care settings, [1] examples of which are given below.

Autoregulation of blood flow is a key feature of the human cerebral vascular system to assure adequate oxygenation and metabolism of the brain under changing physiological conditions. This is essential since due to its high metabolic activity, the brain does not tolerate hypoxia or hypoperfusion. The autoregulation of cerebral blood flow (CBF) provides a steady flow of blood towards the brain by altering vascular resistance through complex myogenic, neurogenic, and metabolic mechanisms. This autoregulatory control mechanism therefore buffers any variations in mean arterial blood pressure (MAP) and cerebral perfusion pressure (CPP) and is effective in a MAP range between approximately $50-150 \mathrm{mmHg}$, defining the lower (LLA) and upper limit of autoregulation (ULA), respectively. This range of intact autoregulation may, however, vary considerably between individuals, and shifts to higher thresholds have been observed in elderly and hypertensive patients. At the blood pressure extremes, i.e. below the LLA and above the ULA, the cerebral vasculature is no longer able to adapt its resistance in response to further blood pressure changes. The clinical consequence is for instance that intraoperative 
hypotension (with MAP values below the LLA) will be poorly tolerated, and might cause ischemic events and postoperative neurological complications. Therefore, besides $\mathrm{ScO}_{2}$, the patient's autoregulatory status might be an important monitoring issue, which could give the clinician important prognostic information on neurologic outcome and allow for adequate therapeutic measures to be taken.

In this regard, the Journal of Clinical Monitoring and Computing (JCMC) welcomes research investigating technologies for the assessment of $\mathrm{ScO}_{2}$ and cerebral autoregulation (CA). In this review, we summarize and discuss papers about monitoring of $\mathrm{ScO}_{2}$ using near infrared spectroscopy (NIRS) as well as publications on CA printed last year in the JCMC.

\section{Near infrared spectroscopy}

The NIRS technology was investigated in several studies published in the journal in 2016. The first two papers comprise volunteer studies analysing the NIRS signal in depth. In the August issue, Colquhoun et al. [2] performed a frequency domain analysis of NIRS signals recorded in 20 volunteers in order to separate the arterial and venous contribution to the signal. The background of their study is the fact that most current commercially available oximetry devices do not discriminate between arterial and venous blood in the investigated sample volume, and assume a fixed ratio of arterial to venous blood varying from a 70:30 ratio to a 80:20 ratio, depending on the device used [3]. Yet, this assumption, which is mainly based on anatomical evidence, may not always be true, and should be more weighted towards arterial haemoglobin saturation, as recently shown [4]. The authors hypothesized that frequency domain analysis of photoplethysmographic (PPG) and NIRS signals may discriminate between arterial and venous blood. In order to alter the contribution of the venous part of the signal, the authors used an impedance threshold device (ITD) in their volunteers, which amplifies the effect of respiratory pressures on blood flow by increasing intrathoracic pressure and thereby might temporarily alter the arterial to venous blood ratio within the brain. $\mathrm{ScO}_{2}$ was measured via a special two-wavelengths portable NIRS device, which is based on spatially resolved spectroscopy techniques. After baseline measurements, the ITD was applied and a second set of measurements was taken. For analysis, the spatially resolved absorbance waveforms were transformed into the frequency domain and relative concentrations of oxygenated and deoxygenated haemoglobin were calculated by using the two wavelengths in seven frequency domains for each individual. While the ITD increased $\mathrm{ScO}_{2}$ by $3.6 \%$ on average, the induced low and high frequency modulations in the NIRS signals could not be exclusively attributed to arterial and venous blood, respectively. Obviously, the low and high frequency components of both the PPG and NIRS waveforms contain contributions from both arterial and venous blood, the relative amounts of which are not known. Of note, since the NIRS waveforms show the same respiratory variations as the arterial pressure [5] or PPG waveforms [6, 7], they might be used to non-invasively determine fluid responsiveness as well, particularly when peripheral perfusion is compromised.

In the April issue, Hirasawa et al. [8] developed an algorithm that eliminates the influence of skin blood flow in the NIRS signal. Against the background of recent literature showing that scalp and skull blood flow (SSBF) may contaminate the NIRS signal traveling through these structures and thus affect $\mathrm{ScO}_{2}$ readings, $[9,10]$ the authors used a headband cuff, which was placed above the superficial temporal artery and inflated repeatedly to $80 \mathrm{mmHg}$ in 12 healthy volunteers in order to suppress SSBF, as verified by laser Doppler flowmetry. To eliminate SSBF influence on the NIRS-derived cerebral oxygenation, most commercial NIRS devices employ two source-detector distances (mostly between 15 and $30 \mathrm{~mm}$ for the short and $40-50 \mathrm{~mm}$ for the long distance) and subtract the signal from the short distance-detector (reflecting superficial tissues) from that of a long-distance (reflecting brain tissue), a method known as spatially resolved NIRS. However, recent literature suggests that this technique does not fully eliminate SSBF influence on the NIRS signal, as shown previously by the group of authors for instance after vasoconstrictor application $[10,11]$. Hence, the authors developed an algorithm with an individual correction factor for extracranial blood flow to isolate cerebral oxygenation from the NIRS signal based on suppressed SSBF. This algorithm was then validated against resting conditions during cerebral activation induced by handgrip exercise and a cognitive task. Both interventions did significantly increase $\mathrm{SSBF}$ and $\mathrm{ScO}_{2}$. Inflation of the headband reduced both SSBF and the original $\mathrm{ScO}_{2}$ under all conditions studied, whereas it did not affect the algorithm-estimated $\mathrm{ScO}_{2}$. The authors conclude that their algorithm with an individual correction factor successfully eliminated the influence of SSBF on the NIRS signal, allowing for measurement of valid (changes in) cerebral oxygenation. The complexity of their approach will however limit its use to special applications such as physiological studies on cerebral activation.

Five more articles are dealing with the impact of $\mathrm{ScO}_{2}$ monitoring on patient management in different clinical settings.

In the April issue, Sorensen et al. [12] report a retrospective study on a ventilation strategy during open abdominal aortic aneurysm repair; in this study, the authors evaluated $\mathrm{ScO}_{2}$ and its relation to end-tidal carbon dioxide tension 
(etCO $\mathrm{CO}_{2}$ ) during the surgery. This study setting is especially interesting because marked hemodynamic changes very rapidly occur during this surgical procedure (clamping and de-clamping of the aorta). This is challenging with regard to hemodynamic and respiratory support as these clamping/de-clamping manoeuvres also induce changes in the patients' metabolism (with reduced cardiac output and metabolism during clamping and an increase in partial pressure of carbon dioxide after reperfusion). The authors analysed 44 patients in whom mechanical ventilation was adjusted according to etCO $\mathrm{CO}_{2}$ and $\mathrm{ScO}_{2}$ was monitored with NIRS. They report that et $\mathrm{CO}_{2}$ and $\mathrm{ScO}_{2}$ were kept constant after aortic clamping by reducing minute ventilation (median $-0.8 \mathrm{~L} \mathrm{~min}$ ). After de-clamping of the aorta, an increase in minute ventilation by a median of $1.8 \mathrm{~L} \mathrm{~min}$ resulted in an increase in $\mathrm{ScO}_{2}$ of $2 \%$, while despite the increase in minute ventilation median etCO $\mathrm{CO}_{2}$ increased by $0.5 \mathrm{kPa}$. From these observations, the authors conclude that $\mathrm{ScO}_{2}$ can be kept within reasonable limits by reducing ventilation by about $1 \mathrm{~L} / \mathrm{min}$ during clamping of the aorta and increasing ventilation by about $2 \mathrm{~L} / \mathrm{min}$ during reperfusion. This rule of thumb adjustment of ventilator management can be further fine-tuned by $\mathrm{ScO}_{2}$ monitoring.

Erdem et al. [13] performed a study (published in the October issue) on the effect of controlled hypotension during elective rhinoplasty on $\mathrm{ScO}_{2}$ assessed using NIRS. The authors included 50 adults in whom controlled hypotension was achieved by using total intravenous anaesthesia and nitroglycerin infusion (if needed). The authors defined "cerebral desaturation" as a decrease in $\mathrm{ScO}_{2}$ of lower than $80 \%$ of individual baseline $\mathrm{ScO}_{2}$ for more than $15 \mathrm{~s}$ and report that this endpoint occurred in 5 out of the 50 patients. Interestingly, none of the episodes of cerebral desaturation was accompanied with a decrease in the peripheral oxygen saturation or the etCO $\mathrm{CO}_{2}$. Therefore, this interesting study demonstrates that NIRS can indicate marked decreases in $\mathrm{ScO}_{2}$ in patients undergoing controlled hypotension even if the peripheral oxygen saturation remains in a normal range.

The relation between hypotension and $\mathrm{ScO}_{2}$ was investigated by Sun et al. (see August issue) [14]. In 45 parturients undergoing combined spinal-epidural (CSE) anaesthesia for Caesarean section, the authors studied if hypotensive episodes (defined as a decrease in systolic blood pressure below $80 \%$ of baseline) could be predicted by a decrease in $\mathrm{ScO}_{2}$. This would be important since hypotension in this setting is frequent (occurring in about $70 \%$ of their patients) and may jeopardize both fetus (hypoxia, acidosis) and parturient (nausea, vomiting, syncope), and common prophylactic measures such as volume loading or vasopressor administration failed to significantly reduce its incidence [15]. The authors prospectively observed $\mathrm{ScO}_{2}$ (the readings of which were blinded for the anaesthetist in charge) and blood pressure (discontinuously every minute) in 45 parturients not receiving any premedication or prophylactic measures to prevent hypotension. A decrease in $\mathrm{ScO}_{2} \geq 5 \%$ from individual baseline values was chosen as threshold for prediction of hypotension. $\mathrm{ScO}_{2}$ decreased significantly more after CSE anaesthesia in parturients developing hypotension as compared to those without hypotension, probably due to a hypotension-induced reduction in CBF. More important, the decrease in $\mathrm{ScO}_{2}$ occurred earlier (about $40 \mathrm{~s}$ ) than did hypotension, a time span sufficient to take corrective therapeutic measures. But how can $\mathrm{ScO}_{2}$ decrease earlier than blood pressure if the decrease in $\mathrm{ScO}_{2}$ is caused by the hypotension? The authors try to explain this by reflex upper-body vasoconstriction and reduction in venous return secondary to CSE anaesthesia, but it could also be due to the higher temporal resolution (seconds) of the $\mathrm{ScO}_{2}$ signals compared to intermittent blood pressure measurements (minutes). Nevertheless, ROC analysis revealed a decrease in $\mathrm{ScO}_{2}$ as a good predictor of hypotension with an optimal threshold value of $4.5 \%$ and a positive predictive value of 0.92 . If NIRS monitoring should be used for prediction or early detection of hypotension (as suggested by the authors) in a broader scale depends on the costs (of disposable sensors) associated with this kind of monitoring. It might also be argued that intensifying blood pressure monitoring towards continuous measurements (such as currently available with several non-invasive methods) [16] will also enable to prevent or reduce the incidence of hypotension significantly as well.

In the October issue, Kerz et al. [17] report an interesting study investigating the correlation of $\mathrm{ScO}_{2}$ measured by continuous-wave NIRS measurements with invasive brain tissue oxygenation measurements $\left(\mathrm{PtiO}_{2}\right)$ in 11 neurosurgical ICU patients. This study approach is interesting because validation data for NIRS - although widely clinically used e.g. in cardiothoracic anaesthesia-are scarce. Interestingly, the authors found very low correlation coefficients for the correlation of NIRS and $\mathrm{PtiO}_{2}$; in addition, the predictive capabilities of NIRS for an $\mathrm{PtiO}_{2}$ of $<15 \mathrm{mmHg}$ were bad (area under the curve of the receiver operating characteristics curve about 0.56). The authors conclude that continuous-wave NIRS does not well correlate with invasively assessed $\mathrm{PtiO}_{2}$ values and that NIRS cannot detect episodes of cerebral ischemia. It has to be emphasized, however, that the authors used the continuous-wave NIRS method that is based on intensity alterations of emitted light. Therefore, results cannot unconditionally be transferred to other more sophisticated NIRS methods (such as frequency-domain or time-domain-based measurements). Furthermore, it has to be stressed that NIRS measures the haemoglobin oxygen saturation of the blood within the arterioles and venules with a signal weighting of approximately 20 versus $80 \%$ or 25 versus $75 \%$, respectively, depending on the device [3]. 
Also in the October issue, a case-series by Brodt et al. [18] was published evaluating changes in cerebral oxygen saturation in 10 patients during transcatheter aortic valve replacement under general anaesthesia. As transcatheter aortic valve replacement is used in cardiovascular high-risk patients and includes rapid-frequency ventricular pacing during valve deployment, patients undergoing this procedure are at risk for decreases in $\mathrm{ScO}_{2}$. The authors report relatively low baseline $\mathrm{ScO}_{2}$ values of $56 \pm 7 \%$ in their high-risk patients. After induction of general anaesthesia, the authors expectedly observed an increase in $\mathrm{ScO}_{2}$. During valve deployment, the mean $\mathrm{ScO}_{2}$ was $49 \pm 13 \%$. In two patients $\mathrm{ScO}_{2}$ decreased more than $20 \%$ compared to baseline values. After valve deployment, $\mathrm{ScO}_{2}$ returned to baseline values in all of the ten patients (this return to baseline, however, took up to $20 \mathrm{~min}$ in three patients (mean $13 \pm 10 \mathrm{~min})$ ). Unfortunately, this case-series does not give details of the functional neurological status of the patients before and after the intervention. Nevertheless, it illustrates that the sudden decrease in cardiac output by rapid-pacing results in a marked transient decrease in $\mathrm{ScO}_{2}$. Strategies to optimize $\mathrm{ScO}_{2}$ prior to valve deployment might improve patient safety during transcatheter aortic valve replacement and should be evaluated in future studies.

In the December issue, an interesting systematic review on the use of NIRS during cardiological procedures by Moerman et al. [19] has been published. The authors hypothesized that NIRS monitoring might help improving patient safety in this group of patients with marked risk for cardiovascular complications. Applying a systematic search strategy to search electronic bibliographic databases the authors identified 11 observational studies (no randomized trial was available) and five case reports on the use of NIRS in patients during cardiological procedures (six studies during electrophysiology for arrhythmias, four studies during pediatric catheterization procedures, one study during transcatheter aortic valve implantations); based on these studies the authors assessed the evidence for the use of NIRS. Based on this limited number of available studies (all of which had a low statistical power) the authors conclude that NIRS provides a very quick representation of $\mathrm{ScO}_{2}$ and that it might identify changes that could not be predicted from standard hemodynamic monitoring during cardiological procedures. Nevertheless, the authors emphasize that the evidence for improved patient outcome is currently not high enough to generally recommend the use of NIRS for all cardiological procedures.

\section{Autoregulation of cerebral blood flow}

In the June issue of the journal, Goettel et al. [20] addressed this issue and investigated the effect of sevoflurane anaesthesia on CA in 133 patients of two different age groups, a younger (age 18-40 years, $n=49$ ) and an elderly cohort (age $\geq 65$ years, $n=84$ ). It is known that volatile anaesthetics impair the CA response in a dose-dependent manner. Therefore, the authors hypothesized that CBF autoregulation would be less effective in older patients as compared to younger study subjects under sevoflurane anaesthesia and expected a shorter autoregulatory plateau due to an increased LLA in older patients. CBF was measured bilaterally by transcranial Doppler (TCD) and blood pressure non-invasively by the finger volume clamp method. Both values were correlated and the linear correlation coefficient Mx taken as a measure of $\mathrm{CBF}$ autoregulation, with a $\mathrm{Mx}$ of 0 indication intact autoregulation and a positive Mx (approaching 1) indicating loss of autoregulation and pressure-driven CBF. In their prospective observational study, they found a LLA of $66 \pm 12 \mathrm{mmHg}$ and $73 \pm 14 \mathrm{mmHg}$ in young and older patients, respectively, but no difference in the ULA $(70 \pm 14 \mathrm{mmHg}$ in older vs. $73 \pm 19 \mathrm{mmHg}$ in younger patients, respectively). Hence, the autoregulatory range was substantially smaller than the expected $100 \mathrm{mmHg}$, and tended to be greater for younger than for older patients $(14 \pm 10 \mathrm{mmHg}$ vs. $10 \pm 9 \mathrm{mmHg})$. Furthermore, Mx was significantly higher in older compared to younger patients, indicating that $\mathrm{CBF}$ autoregulation was less effective in the elderly. The authors conclude that the autoregulatory plateau is shortened substantially in both young and older patients under sevoflurane anaesthesia with approximately 1 MAC as compared to awake subjects, probably due to its vasodilator effects. However, other factors on CA such as patient comorbidity, carbon dioxide levels, cerebral metabolism, and vasoactive agents cannot be excluded. Remarkably, the LLA and ULA, as well as the autoregulatory range were not influenced by the age of anaesthetized patients. The results imply that patients under general anaesthesia are less protected by CA and may be more susceptible to cerebral ischemia or edema.

In an accompanying editorial, Moerman and Absalom [21] point out some weaknesses of the abovementioned study, including the fact that in the majority of their patients (89\%), the LLA and/or ULA were not reached, mainly because major fluctuations in blood pressure were prevented. Nevertheless, they acknowledge the importance of the study findings that sevoflurane may alter the position and shape of the CA curve and the implications thereof for an individualized perioperative hemodynamic management.

CA is certainly important in traumatic brain injury (TBI), which may be associated with intracranial hypertension. In the December issue, Kim et al. [22] report the results of their automatic data monitoring for CA. They developed an integrated platform for acquiring and evaluating data necessary for developing predictive 
models and collected pressure data from 29 TBI patients admitted to their ICU. Subsequently, they used the established pressure reactivity index (PRx), which is based on the assumption that intracranial pressure (ICP) should not directly correlate with arterial blood pressure, and found that it can predict intracranial hypertensive events (defined as ICP increases above $25 \mathrm{mmHg}$ for $>5 \mathrm{~min}$ ) in the hour preceding the event. The accuracy of the prediction based on a certain PRx threshold (i.e. $>0.8$ ) was, however, rather low. Furthermore, it has to be shown in future studies if these methods based on retrospective analyses of intracranial hypertensive events that had already occurred can be transferred to predict and probably prevent such events.

In the October issue, Montgomery et al. [23] performed a secondary analysis on a porcine dataset (containing NIRS and systemic blood pressure data) to investigate data clustering methods as a technique for determining the LLA. This way they question the traditional approach of using binned data to assess CA functionality. A noninvasive method using NIRS technology instead of TCD was used as reference. For this, the $\mathrm{ScO}_{2}$ and MAP values were correlated, and, similar to the above mentioned $\mathrm{Mx}$, the resultant Pearson correlation coefficient COx will be near zero in case of intact CA but around 1 in case of impaired CA. Binning the data in pressure increments of e.g. $5 \mathrm{mmHg}$ allows to visually determine the LLA and ULA thresholds, by identifying the step increase in COx. As alternative technique of differentiating the intact and impaired CBF autoregulation zones, the authors developed a novel model using two automated data clustering methods based on historical raw (unbinned) data from porcine experiments. For this purpose, seven pigs had been exposed to different interventions including hyperand hypoventilation, lung recruitment manoeuvres, acute hypoxia, and haemorrhagic shock. They used a rather high COx threshold of 0.5 to differentiate intact from impaired $\mathrm{CA}$ in order to reduce the influence of noisy values tending to zero. Subsequently, they compared both methods of determining the LLA and found a good agreement. Both of their clustering methods revealed very distinct LLA thresholds (while ULA threshold could not be determined due to lack of data), which were comparable albeit slightly lower than those derived from the traditionally binned data algorithm. The authors conclude that their new method of determining the LLA of CA is feasible and may be considered an alternative method in continuous NIRS-based CA monitoring, particularly in noisy environments (in terms of data purity) such as those frequently encountered in clinical practice. Furthermore, their methods might also apply to other correlation-based methods of determining CA thresholds, such as the Mx or PRx modalities mentioned earlier.

\section{Summary}

In summary, the above-mentioned studies on $\mathrm{ScO}_{2}$ and CA present an update in current functional cerebral monitoring. It remains to be shown if the findings related to signal processing will find their way to clinical applicability, and if the clinical findings presented here will be reproduced in larger clinical trials. Nevertheless, the JCMC has established its leading role as platform for research related to the topics of $\mathrm{ScO}_{2}$ and CA monitoring.

\section{Compliance with ethical standards}

Conflict of interest TWLS and BS have no conflicts of interest to declare.

Research involving human participants and/or animals Not applicable. This is a review article not including human participants and/or animals.

Informed consent Not applicable. This is a review article not including human participants and/or animals.

Open Access This article is distributed under the terms of the Creative Commons Attribution 4.0 International License (http:// creativecommons.org/licenses/by/4.0/), which permits unrestricted use, distribution, and reproduction in any medium, provided you give appropriate credit to the original author(s) and the source, provide a link to the Creative Commons license, and indicate if changes were made.

\section{References}

1. Scheeren TW, Schober P, Schwarte LA. Monitoring tissue oxygenation by near infrared spectroscopy (NIRS): background and current applications. J Clin Monit Comput. 2012;26(4):279-87. doi:10.1007/s10877-012-9348-y.

2. Colquhoun DA, Naden K, Thiele RH. Frequency domain analysis of cerebral near infrared spectroscopy signals during application of an impedance threshold device in spontaneously ventilating volunteers. J Clin Monit Comput. 2016;30(4):38998. doi:10.1007/s 10877-015-9729-0.

3. Scheeren TW, Bendjelid K. Journal of clinical monitoring and computing 2014 end of year summary: near infrared spectroscopy (NIRS). J Clin Monit Comput. 2015;29(2):217-20. doi:10.1007/s10877-015-9689-4.

4. Sorensen H, Secher NH, Rasmussen P. A note on arterial to venous oxygen saturation as reference for NIRS-determined frontal lobe oxygen saturation in healthy humans. Front Physiol. 2013;4:403. doi:10.3389/fphys.2013.00403.

5. Mathis MR, Schechtman SA, Engoren MC, Shanks AM, Thompson A, Kheterpal S, Tremper KK. Arterial pressure variation in elective noncardiac surgery: identifying reference distributions and modifying factors. Anesthesiology. 2017. doi:10.1097/ALN.0000000000001460.

6. Addison PS. A review of signal processing used in the implementation of the pulse oximetry photoplethysmographic fluid 
responsiveness parameter. Anesth Analg. 2014;119(6):1293306. doi:10.1213/ANE.0000000000000392.

7. Alian AA. Anesthesiologist as physiologist: discussion and examples of clinical waveform analysis. Anesth Analg. 2017;124(1):154-166. doi:10.1213/ANE.0000000000001468.

8. Hirasawa A, Kaneko T, Tanaka N, Funane T, Kiguchi M, Sorensen H, Secher NH, Ogoh S. Near-infrared spectroscopy determined cerebral oxygenation with eliminated skin blood flow in young males. J Clin Monit Comput. 2016;30(2):243-50. doi:10.1007/s10877-015-9709-4.

9. Davie SN, Grocott HP. Impact of extracranial contamination on regional cerebral oxygen saturation: a comparison of three cerebral oximetry technologies. Anesthesiology. 2012;116(4):83440. doi:10.1097/ALN.0b013e31824c00d7.

10. Sorensen H, Secher NH, Siebenmann C, Nielsen HB, KohlBareis M, Lundby C, Rasmussen P. Cutaneous vasoconstriction affects near-infrared spectroscopy determined cerebral oxygen saturation during administration of norepinephrine. Anesthesiology. 2012;117(2):263-70. doi:10.1097/ALN.0b013e3182605afe.

11. Sorensen H, Rasmussen P, Sato K, Persson S, Olesen ND, Nielsen HB, Olsen NV, Ogoh S, Secher NH. External carotid artery flow maintains near infrared spectroscopy-determined frontal lobe oxygenation during ephedrine administration. Br J Anaesth. 2014;113(3):452-8. doi:10.1093/bja/aet481.

12. Sorensen H, Nielsen HB, Secher NH. Near-infrared spectroscopy assessed cerebral oxygenation during open abdominal aortic aneurysm repair: relation to end-tidal $\mathrm{CO}_{2}$ tension. J Clin Monit Comput. 2016;30(4):409-15. doi:10.1007/s10877-015-9732-5.

13. Erdem AF, Kayabasoglu G, Tas Tuna A, Palabiyik O, Tomak Y, Beyaz SG. Effect of controlled hypotension on regional cerebral oxygen saturation during rhinoplasty: a prospective study. J Clin Monit Comput. 2016;30(5):655-60. doi:10.1007/ s10877-015-9768-6.

14. Sun S, Liu NH, Huang SQ. Role of cerebral oxygenation for prediction of hypotension after spinal anesthesia for caesarean section. J Clin Monit Comput. 2016;30(4):417-21. doi:10.1007/ s10877-015-9733-4.
15. Arndt JO, Bömer W, Krauth J, Marquart B. Incidence and time course of cardiovascular side effects during spinal anesthesia after prophylactic administration of intravenous fluids or vasoconstrictors. Anesth Analg. 1998;87(2):347-354.

16. Teboul JL, Saugel B, Cecconi M, De Backer D, Hofer CK, Monnet X, Perel A, Pinsky MR, Reuter DA, Rhodes A, Squara P, Vincent JL, Scheeren TW. Less invasive hemodynamic monitoring in critically ill patients. Intensive Care Med. 2016;42(9):1350-9. doi:10.1007/s00134-016-4375-7.

17. Kerz T, Beyer C, Huthmann A, Kalasauskas D, Amr AN, Boor S, Welschehold S. Continuous-wave near-infrared spectroscopy is not related to brain tissue oxygen tension. J Clin Monit Comput. 2016;30(5):641-7. doi:10.1007/s10877-015-9755-y.

18. Brodt J, Vladinov G, Castillo-Pedraza C, Cooper L, Maratea E. Changes in cerebral oxygen saturation during transcatheter aortic valve replacement. J Clin Monit Comput. 2016;30(5):649-53. doi:10.1007/s10877-015-9758-8.

19. Moerman A, Meert F, De Hert S. Cerebral near-infrared spectroscopy in the care of patients during cardiological procedures: a summary of the clinical evidence. J Clin Monit Comput. 2016;30(6):901-9. doi:10.1007/s10877-015-9791-7.

20. Goettel N, Patet C, Rossi A, Burkhart CS, Czosnyka M, Strebel SP, Steiner LA. Monitoring of cerebral blood flow autoregulation in adults undergoing sevoflurane anesthesia: a prospective cohort study of two age groups. J Clin Monit Comput. 2016;30(3):25564. doi:10.1007/s10877-015-9754-z.

21. Moerman A, Absalom AR. You can't manage what you don't measure. J Clin Monit Comput. 2016;30(3):253-4. doi:10.1007/ s10877-015-9797-1.

22. Kim N, Krasner A, Kosinski C, Wininger M, Qadri M, Kappus Z, Danish S, Craelius W. Trending autoregulatory indices during treatment for traumatic brain injury. J Clin Monit Comput. 2016;30(6):821-31. doi:10.1007/s10877-015-9779-3.

23. Montgomery D, Addison PS, Borg U. Data clustering methods for the determination of cerebral autoregulation functionality. J Clin Monit Comput. 2016;30(5):661-8. doi:10.1007/ s10877-015-9774-8. 